\title{
TACHINIDAE (DIPTERA) FROM THE CALDERA DE TABURIENTE NATIONAL PARK, LA PALMA (CANARY ISLANDS)
}

\author{
H.-P. Tschorsnig*, T. Domingo-Quero** and M. A. Alonso-Zarazaga**
}

\begin{abstract}
Data on distribution, abundance and phenology of Tachinidae (Diptera) from the Caldera de Taburiente National Park on La Palma are given, based on systematic Malaise trap and yellow pan trap samples between August 1999 and July 2001. The occurrence of 22 species is proved; all of them were already known from the Canary Islands, but two species (Gonia quadrisetosa and Phytomyptera vaccinii) are recorded from La Palma for the first time. The present paper is a result of the project "Inventory and study of the invertebrate fauna of the Caldera de Taburiente National Park".
\end{abstract}

Key words: Diptera, Tachinidae, Caldera de Taburiente National Park, La Palma, Canary Islands.

\section{RESUMEN}

\section{Tachinidae (Diptera) del Parque Nacional de La Caldera de Taburiente} en La Palma (Islas Canarias)

Se presentan datos de distribución, abundancia y fenología de Tachinidae (Diptera), obtenidos en el Parque Nacional de la Caldera de Taburiente en La Palma con trampa Malaise y trampa amarilla entre agosto de 1999 y julio de 2001. Han sido recolectadas 22 especies, todas ya conocidas en las Islas Canarias, pero dos especies (Gonia quadrisetosa y Phytomyptera vaccinii) son citas nuevas para La Palma. El presente artículo es un resultado del proyecto "Inventario y estudio de la fauna invertebrada del Parque Nacional de la Caldera de Taburiente".

Palabras clave: Diptera, Tachinidae, Parque Nacional de La Caldera de Taburiente, La Palma, Islas Canarias.

* Staatliches Museum für Naturkunde. Rosenstein 1. 70191 Stuttgart (Germany). E-mail: tschorsnig.smns@naturkundemuseum-bw.de

** Departamento de Biodiversidad. Museo Nacional de Ciencias Naturales de Madrid. C/ José Gutierrez Abascal, 2. 28006 Madrid (Spain). E-mail: monaz39@mncn.csic.es 


\section{Introduction}

Tachinidae is a very large family of Diptera; nearly 1800 species are currently known from the Palaearctic region. The larvae develop as parasitoids in various host orders of insects (mostly Lepidoptera, but also Hymenoptera, Coleoptera, Hemiptera, Orthoptera, and some others), rarely also in some other Arthropoda.

The Tachinidae of the Canary Islands were treated 20 years ago by Báez et al. (1986). All 22 species of the present investigation were already recorded from the Canary Islands, but most of them were known before from La Palma only by a very low number of specimens and some of them were based only on very old single records. Two species (Gonia quadrisetosa, and Phytomyptera vaccinii) are recorded from La Palma for the first time. On the basis of the same investigation, an additional four species (Exorista kugleri, Ceracia mucronifera, Aplomya confinis and Cylindromyia brassicaria) were already indicated as new in the paper of Domingo-Quero et al. (2003).

The present paper provides data on the distribution and phenology of Tachinidae from the Caldera de Taburiente National Park on La Palma, based on systematic Malaise trap and yellow pan trap samples. It is a result of the project "Convenio de Cooperación Parques Nacionales - CSIC Inventario y estudio de la fauna invertebrada del Parque Nacional de la Caldera de Taburiente". General information on this project and its first results is provided by Domingo-Quero et al. (2003).

\section{Material and Methods}

The material was systematically collected at five localities (see below) in a Malaise trap, type Townes (MT) and a combined yellow pan trap (YT) by T. Domingo-Quero between August 1999 and July 2001. The traps were emptied at intervals of between 4 and 15 days (average of 7.75 days). Only two specimens were obtained by other methods (netted from flowering plants). The yellow pan trap ("Moericke trap") was placed under the middle wall of each Malaise trap. For further details see Domingo-Quero et al. (2003).

List of localities (in order of ascending altitudes):

Playa de Taburiente, 750 m, UTM 28RBS1980-1

Barranco de Las Traves, 1068 m, UTM 28RBS1780-2

Lomo de las Chozas, 1277 m [1299 m during the year 1999], UTM 28RBS2077-3
Roque de la Cumbrecita ("parcela de los Helianthemum"), 1377 m, UTM 28RBS2177-1

Veta de Tajodeque, $2100 \mathrm{~m}$, UTM 28RBS1682-4 (a single record, not trapped)

Roque de los Muchachos ("parcela del Morro de la Cebolla"), 2250 m, UTM 28RBS2084-3

Pico de la Cruz, 2345 m, UTM 28RBS2183-1 (a single record, not trapped).

In total, there are 1480 collected specimens. The material is preserved in the Museo Nacional de Ciencias Naturales Madrid (Spain), except for 165 duplicate specimens of a few species which are stored in the Naturkundemuseum Stuttgart (Germany).

\section{Species list}

The arrangement of subfamilies, genera and species follows Herting \& Dely-Draskovits (1993). The data are sorted by locality (in order of ascending altitude) and date. The date means the day when a trap was emptied. Information on distribution in the Canary Islands is based on Báez et al. (1986), but updated with unpublished material. No host records of Tachinidae are known from La Palma; the general information on hosts is derived from a database on Palaearctic host-parasitoid records of tachinids which is currently being compiled by the first author.

\section{Subfamily Exoristinae}

Exorista segregata (Rondani, 1859)

MAterial (Total 17 MT, 30 YT). Playa de Taburiente: 18.X.1999 (1 ㅇ YT), 10.I.2000 (1 우 MT), 20.III.2000 (1 우 MT), 4.VII.2000 (1 $\sigma^{7}$ YT), 3.VIII.2000 (1 $\sigma^{7} 1$ \& $\left.\mathrm{YT}\right)$, 7.VIII.2000 (10 MT; $60^{\pi} \sigma^{7} 1$ 우 YT), 14.VIII.2000 (1 우 YT), 22.VIII.2000 (1 $\sigma^{7}$ YT). - Barranco de Las Traves: 3.VIII.1999

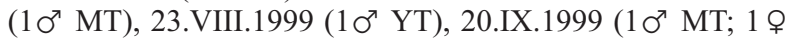
YT), 30.V.2000 (1 ㅇ MT), 13.VI.2000 (1 ○ MT), 14.VIII.2000 (1 우 YT). - Lomo de las Chozas: 22.IX.1999 $\left(20^{x} 0^{x} 3\right.$ 우 우 YT), 20.X.1999 (2우우 MT; $\left.10^{\pi} \mathrm{YT}\right)$, 24.XI.1999 (1 우 MT), 8.XII.1999 (1 $\sigma^{\top} 1$ 우 MT), 22.III.2000 (10 YT), 26.IV.2000 (1 $\left.\sigma^{\pi} \mathrm{MT}\right), 10 . \mathrm{V} .2000$ (1 $\left.\sigma^{\pi} \mathrm{MT}\right)$, 26.VII.2000 (1 우 MT), 16.VIII.2000 (1 $0^{\pi}$ MT; 1 우 YT), 24.VIII.2000 (1 \& YT). - Roque de la Cumbrecita: 7.IX.1999 (1 주 1 우 YT), 22.IX.1999 (1 우 YT), 10.XI.1999 (1 우 YT), 23.II.2000 (1 ㅇ MT). - Roque de los Muchachos: 25.VIII.2000 (1 ㅇ YT).

Distribution. Mediterranean, Central Asia. - Canary Islands: Tenerife, La Palma.

Hosts. A wide range of lepidopteran hosts of many families. No host record from the Canary Islands. 
Exorista sorbillans (Wiedemann, 1830)

Material (Total 1 YT). Playa de Taburiente: 22.VIII.2000 (1 우 YT).

Distribution. Widespread in the Mediterranean, Central Asia, Japan, Afrotropical and Oriental Regions. - Canary Islands: Tenerife, Gran Canaria, La Gomera, La Palma, El Hierro.

Hosts. Lepidoptera of many families. Host records from the Canary Islands: Danaus chrysippus Linnaeus and D. plexippus Linnaeus (Danaidae), Catopsilia florella Fabricius (Pieridae), Cornutiplusia circumflexa Linnaeus (Noctuidae), Acherontia atropos Linnaeus and Hyles livornica Esper (Sphingidae) (Báez et al., 1986; Tschorsnig \& Herting, 1997; Tschorsnig, in press; unpublished).

\section{Exorista kugleri Mesnil, 1960}

Material (Total 2 YT). Barranco de Las Traves: 5.IX.1999 (1 ㅇ YT). - Lomo de las Chozas: 16.VIII.2000 (1 ㅇ YT).

Distribution. Known from Tunesia, Palestine, Tadzhikistan. - Canary Islands: Tenerife, Gran Canaria, La Gomera, La Palma.

Hosts. Unknown.

\section{Chetogena acuminata Rondani, 1859}

MATERIAL (Total 19 MT, 84 YT). Playa de Taburiente: 18.X.1999 (10 $0^{\top}$ MT), 3.IV.2000 (1 $\sigma^{\top}$ MT), 10.IV.2000 (1 우

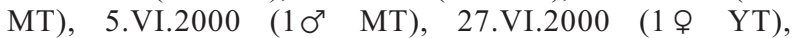
24.VII.2000 (1 $10^{7}$ YT), 3.VIII.2000 (2 우 우 YT), 7.VIII.2000 (10 MT), 22.VIII.2000 (10 MT). - Barranco de Las Traves: 3.VIII.2000 (1 ㅇ YT). - Lomo de las Chozas: 22.IX.1999 (1우 MT; $2 \sigma^{\top} \sigma^{\pi} 5$ 우 우 YT), 20.X.1999 (1우 MT), 10.XI.1999 (1 $\sigma^{\pi}$ MT), 1.VI.2000 (1 $\sigma^{\pi} 1$ 우 MT; $2 \sigma^{\pi} \sigma^{\pi}$ YT), 21.VI.2000 (1 우 MT), 6.VII.2000 (1 $\sigma^{7}$ YT), 26.VII.2000 (1 \& YT), 16.VIII.2000 (10 MT; $90^{7} 0^{7}$ 13 우 우 YT), 24.VIII.2000 (1 $\sigma^{\top} 3$ 우 우 YT). - Roque de la Cumbrecita: 7.IX.1999 (2우 우 YT), 22.IX.1999 (1 ㅇ YT), 10.V.2000 (1우 MT), 1.VI.2000 (2우 우 MT; 1 우 $\mathrm{YT}$ ), 21.VI.2000 (2우우 MT), 19.VII.2000 (1우 YT), 16.VIII.2000 (1 \& YT). - Roque de los Muchachos: 27.VII.2000 (3 $\sigma^{x} \sigma^{x}$ YT), 4.VIII.2000 (9 $\sigma^{x} \sigma^{x}$ YT), 31.V.2001 (2우 우 YT), 6.VI.2001 (6우 우 YT), 14.VI.2001 (1 우 MT; 4 우 우 YT), 20.VI.2001 (2우우 YT), 28.VI.2001

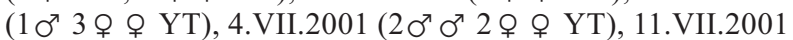
(20 ๙ $\sigma^{\pi T}$ ).

DistRIBUTION. Mediterranean, on seashores northwards to South England; Central Asia, Japan. - Canary Islands: Tenerife, Gran Canaria, La Gomera, La Palma, El Hierro, Fuerteventura, Lanzarote.

Hosts. Larvae of Tenebrionidae (Coleoptera). No host record from the Canary Islands.
Ceracia mucronifera Rondani, 1865

Material (Total 94 MT, 8 YT). Playa de Taburiente: 30.V.2000 (1 ᄋ MT), 5.VI.2000 (1 $0^{7}$ MT), 19.VI.2000 (1 $0^{7}$ MT), 27.VI.2000 (1 우 MT), 17.VII.2000 (1 우 MT), 7.VIII.2000 (10 MT), 22.VIII.2000 (1 $\sigma^{\pi} \mathrm{YT}$ ), 28.VIII.2000 (10 $0^{7} 1$ 우 MT). - Barranco de Las Traves: 3.VIII.1999 (6 우 우 MT), 10.VIII.1999 (5 우 우 MT; 1 우 YT), 23.VIII.1999 (20 ه ه 7 우 우 MT; $10^{\pi} 4$ 우 우 YT), 5.IX.1999 (4우 우 MT), 20.IX.1999 (10 1 우 MT; 1 우 YT), 5.VI.2000 (1우 MT), 13.VI.2000 (10 MT), 4.VII.2000 (1 ᄋ MT), 10.VII.2000

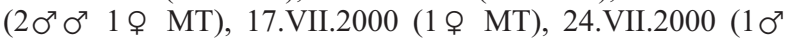
2 우 우 MT), 7.VIII.2000 (3우 우 MT), 14.VIII.2000 (3우 우 MT), 22.VIII.2000 (1 $\sigma^{7} 10$ 우 우 MT), 28.VIII.2000 (1 $\left.\sigma^{7} \mathrm{MT}\right)$, 4.IX.2000 (20 $\sigma^{\top} \sigma^{\top} 1$ 우 MT), 18.IX.2000 (5 $5 \sigma^{\top} \sigma^{\top} 2$ 우 우 MT). Lomo de las Chozas: 22.IX.1999 (10 $0^{\top}$ MT). - Roque de la Cumbrecita: 12.VIII.1999 (10 3 우 우 MT), 22.IX.1999 (3우 우 MT), 20.X.1999 (1우 MT), 10.XI.1999 (1 우 MT), 26.IV.2000 (1 ᄋ MT), 21.VI.2000 (1 오 MT), 19.VII.2000 (1 $0^{\pi}$ MT), 26.VII.2000 (10 1 우 MT), 16.VIII.2000 (2 우 $\mathrm{MT}$ ), 24.VIII.2000 (1 $1 \sigma^{\pi} 1$ 우 MT), 6.IX.2000 (2 $\sigma^{\pi} \sigma^{\pi} 1$ 우 MT).

Distribution. Mediterranean to Transcaucasia. Canary Islands: Tenerife, La Gomera, Lanzarote, La Palma.

Hosts. Acrididae (Orthoptera). Host record from the Canary Islands (by Enderlein, 1930, as Pamphagophaga gomerana Enderlein): Acrostira bellamyi Uvarav.

\section{Nemorilla maculosa (Meigen, 1824)}

Material (Total 5 MT, 56 YT). Playa de Taburiente:

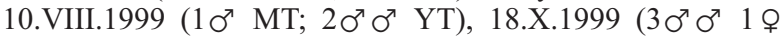
YT), 6.III.2000 (1 ᄋ MT), 17.VII.2000 (1 ㅇ MT), 3.VIII.2000 (30" $\sigma^{7} 1$ 우 YT), 7.VIII.2000 (1 $10^{7} 1$ 우 YT), 22.VIII.2000 (1 $\sigma^{7}$ 1 우 YT), 25.IX.2000 (1 $\sigma^{\top}$ MT). - Barranco de Las Traves: 3.VIII.1999 (1 $\sigma^{7}$ YT), 23.VIII.1999 (1 $10^{7} 1$ \& YT), 5.IX.1999 (10 Y YT), 19.VI.2000 (1 $0^{\pi} 1$ ㅇ YT), 27.VI.2000 (1 ㅇ YT), 10.VII.2000 (1 \& YT), 24.VII.2000 (1 \& YT), 3.VIII.2000 (30 $\sigma^{\pi}$ YT), 7.VIII.2000 (1 \& YT), 18.IX.2000 (1 $\sigma^{\pi}$ YT). Roque de la Cumbrecita: 19.VII.2000 (1 $\sigma^{7}$ MT), 6.IX.2000 (2 우 우 YT). - Roque de los Muchachos: 27.VII.2000 (3우 우 YT), 4.VIII.2000 (1 $\sigma^{\pi} 14$ ㅇ ㅇ YT), 25.VIII.2000 (6 $0^{\pi} \sigma^{\top}$ YT), 31.VIII.2000 (1 $\sigma^{7}$ YT), 7.IX.2000 (1 $\left.10^{\top} \mathrm{YT}\right)$.

Distribution. Palaearctic. - Canary Islands: Tenerife, Gran Canaria, La Gomera, La Palma, El Hierro, Fuerteventura.

Hosts. Many species of Microlepidoptera and a few Macrolepidoptera. No host record from the Canary Islands.

\section{Aplomya confinis (Fallén, 1820)}

Material (Total 30 MT, 6 YT). Playa de Taburiente: 22.VIII.2000 (1 $\sigma^{7}$ MT). - Barranco de Las Traves: 3.VIII.1999 (1 ᄋ YT), 10.VIII.1999 (1 $\sigma^{7}$ MT), 1.V.2000 (1 $\left.\sigma^{7} \mathrm{MT}\right)$,

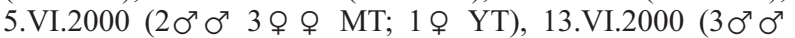
3 우 우 $\mathrm{MT}$; $\left.1 \sigma^{\pi} \mathrm{YT}\right), 19 . \mathrm{VI} .2000$ (20 $\sigma^{\pi} 2$ 우 우 $\left.\mathrm{MT}\right)$, 
27.VI.2000 (10 MT; 1 우 YT), 10.VII.2000 (1 우 MT), 17.VII.2000 (1 우 MT), 24.VII.2000 (1 $\left.\sigma^{\pi} \mathrm{YT}\right)$, 7.VIII.2000 (2우 우 MT), 22.VIII.2000 (1 우 YT). - Lomo de las Chozas: 19.VII.2000 (1 \& MT). - Roque de la Cumbrecita: 26.IV.2000 (1 우 MT), 6.VII.2000 (1 우 MT). - Roque de los Muchachos: 4.VIII.2000 (1 옥 MT), 6.VI.2001 (1 우 MT), 20.VI.2001 (1 우 MT), 28.VI.2001 (1 ㅇ MT).

Distribution. Palaearctic. - Canary Islands: Tenerife, La Gomera, El Hierro, La Palma.

Hosts. Specific parasitoid of Lycaenidae (Lepidoptera). No host record from the Canary Islands.

Pales cyanea (Macquart, 1839)

Material (Total 6 MT, 21 YT). Playa de Taburiente: 6.III.2000 (10 MT), 3.IV.2000 (10 MT), 1.V.2000 (1 우 YT), 7.VIII.2000 (2 ᄋ 우 YT), 14.VIII.2000 (1 ㅇ YT), 22.VIII.2000 (1 우 YT), 18.IX.2000 (2 $0^{\top} \sigma^{7} 1$ 우 YT). - Lomo de las Chozas: 22.IX.1999 (1 $\sigma^{\pi}$ YT), 26.IV.2000 (1 MT), 24.V.2000 (1 웅 YT). - Roque de la Cumbrecita: 10.V.2000 (1 9 MT), 24.V.2000 (1 \& MT). - Roque de los Muchachos: 4.VIII.2000

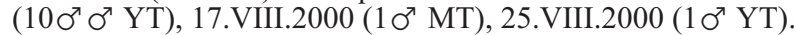

Distribution. Endemic to the Canary Islands (Tenerife, La Gomera, La Palma).

Hosts. Reared from Anomis erosa Huebner (Lepidoptera, Noctuidae) on La Gomera (Leipnitz \& Tschorsnig, 2005).

\section{Gonia quadrisetosa Becker, 1908}

Material (Total 4 MT, 1 YT). Roque de los Muchachos: 21.III.2001 (1 우 YT), 10.IV.2001 (1 $0^{7} 1$ 우 MT), 18.IV.2001 (1 우 MT), 31.V.2001 (1우 MT).

Distribution. Endemic to the Canary Islands (Tenerife, first record for La Palma).

Hosts. Unknown.

REMARKS. This species is very similar to Gonia ornata Meigen, 1826. Mesnil (1956) treated G. quadrisetosa as a variety of $G$. ornata, Báez et al. (1986) understood it as a subspecies, but Herting \& Dely-Draskovits (1993) gave it species rank.

\section{Pseudogonia fasciata (Wiedemann, 1819)}

Material (Total 5 MT, 3 YT, 1 on Adenocarpus viscosus). Playa de Taburiente: 15.XI.1999 (1 우 MT), 18.IX.2000 (1 우 MT). - Roque de la Cumbrecita: 26.VII.2000 (1 ᄋ YT). Roque de los Muchachos: 25.VIII.2000 (1 우 MT), 14.IX.2000 (1 우 YT), 21.IX.2000 (1 우 YT), 10.IV.2001 (1우 MT), 31.V.2001 (1 \& MT). - Pico de la Cruz: 16.IX.1999 (1 우 on Adenocarpus viscosus).

Distribution. Described from the Cape Province in South Africa. Otherwise known from the Canary Islands
(Tenerife, Gran Canaria, La Gomera, La Palma) and northeastern Spain.

Hosts. Unknown.

Subfamily Tachininae

Tachina canariensis (Macquart, 1839)

Material (Total 17 MT, 4 YT). Playa de Taburiente: 8.XI.1999 (10 MT), 3.IV.2000 (1 우 MT), 24.IV.2000 (1 ㅇ MT), 7.VIII.2000 (1 우 MT). - Lomo de las Chozas: 22.III.2000 (1 \& MT). - Roque de los Muchachos: 27.VII.2000 (1 \& YT), 17.VIII.2000 (1 \& MT), 25.VIII.2000 (10" YT), 14.IX.2000 (10 MT), 21.IX.2000 (1 $\sigma^{7}$ YT), 17.X.2000 (10 MT), 4.IV.2001 (1 ㅇ MT), 18.IV.2001 (1 ○ MT), 9.V.2001 (1 ㅇ MT), 17.V.2001 (1 우 MT), 23.V.2001 (2우 우 MT), 6.VI.2001 (2우 우 MT), 28.VI.2001 (1 Q YT), 11.VII.2001 (1 ○ MT).

Distribution. Endemic to the Canary Islands (Tenerife, Gran Canaria, La Gomera, La Palma, El Hierro).

Hosts. Unknown.

REMARKS. Very closely related to Tachina casta (Rondani, 1859). Tachina canariensis was erroneously omitted in the catalogue of Herting \& Dely-Draskovits (1993).

\section{Linnaemya soror Zimin, 1954}

Material (Total 3 MT, 1 YT). Playa de Taburiente: 17.IV.2000 (1 우 MT). - Lomo de las Chozas: 21.VI.2000 (1 우 MT). Roque de los Muchachos: 14.VI.2001 (1 \& MT), 4.VII.2001 (1 \& YT).

Distribution. Mediterranean, Near East, Central Asia, southern Siberia. - Canary Islands: Tenerife, Gran Canaria, La Gomera, La Palma, El Hierro, Fuerteventura, Lanzarote.

Hosts. Unknown.

REMARKS. Before the present investigation there was only one record from La Palma, about one hundred years old (Becker, 1908).

\section{Macquartia tessellum (Meigen, 1824)}

Material (Total 5 MT, 2 YT). Barranco de Las Traves: 20.III.2000 (1 우 MT). - Lomo de las Chozas: 9.II.2000 (1 우 MT). - Roque de los Muchachos: 27.VII.2000 (1 우 YT), 17.VIII.2000 (1 \& MT), 31.VIII.2000 (1 \& YT), 6.VI.2001 (1 ㅇ MT), 20.VI.2001 (1 ㅇ MT).

Distribution. Palaearctic, except northern countries and East Asia. - Canary Islands: Tenerife, La Palma, El Hierro.

Hosts. Larvae of various Chrysomelidae (Chrysolina, Phytodecta, Colaphellus, Entomoscelis). No host record from the Canary Islands. 
REMARKS. Before the present investigation there was only one record from La Palma, about one hundred years old (Becker, 1908).

\section{Phytomyptera vaccinii Sintenis, 1897}

Material (Total 7 MT, 1 YT). Playa de Taburiente: 20.III.2000 (10 2 우 우 MT), 4.VII.2000 (10 $\left.\sigma^{7} \mathrm{MT}\right), 17 . \mathrm{VII} .2000$ (1우 MT), 7.VIII.2000 (10 1 우 MT; 1 ㅇ YT).

Distribution. Europe, Near East. - Canary Islands: Tenerife, first record for La Palma.

Hosts. Gracilariidae (Caloptilia) and Tortricidae (Prays, Epinotia) (Microlepidoptera). No host record from the Canary Islands.

REMARKS. In Báez et al. (1986) recorded under the synonymous name $P$. gracilariae (Hering, 1926).

\section{Siphona seyrigi Mesnil, 1960}

Material (Total 410 MT, 192 YT, 1 on flowers). Playa de Taburiente: 10.VIII.1999 (1 우 MT), 18.X.1999 (2우 우 MT), 3.I.2000 (1 $\sigma^{7}$ MT), 10.I.2000 (1 ㅇ MT), 7.II.2000 (3 $\sigma^{7} \sigma^{7}$ MT), 21.II.2000 (8 $8 \sigma^{7} \sigma^{7} 3$ 우 우 MT), 6.III.2000 (9 $9 \sigma^{\top} \sigma^{\top} 1$ 우 MT), 20.III.2000 (1울 MT), 3.IV.2000 (1 $\sigma^{\top} 12$ 우 우 MT), 10.IV.2000 (7 $7 \sigma^{7} 5$ 우 우 MT), 17.IV.2000 (7 $\sigma^{7} \sigma^{\pi} 7$ 우 우 MT; 1 우 YT), 24.IV.2000 (30 $\sigma^{\pi} 8$ 우 우 MT; $1 \sigma^{\pi} 1$ 우 YT),

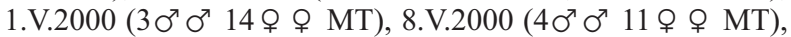
15.V.2000 (5 $5 \sigma^{7} \sigma^{7} 4$ 우 우 MT), 30.V.2000 (6 $6 \sigma^{7} \sigma^{7} 10$ 우 우 MT;

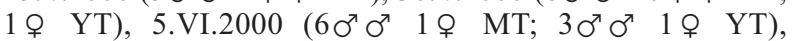

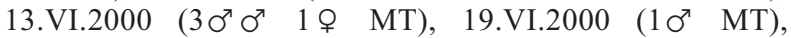
4.VII.2000 (1 $\sigma^{\pi}$ MT), 24.VII.2000 (1 우 MT; $1 \sigma^{\pi}$ YT), 3.VIII.2000 (5 $5 \sigma^{7} \sigma^{7} 1$ 우 YT), 7.VIII.2000 (1 $\sigma^{7} 3$ 우 우 MT), 14.VIII.2000 (1 $\sigma^{\pi} 1$ ㅇ MT; 1 우 YT), 22.VIII.2000 ( $2 \sigma^{\pi} \sigma^{\pi} 1$ ㅇ MT), 28.VIII.2000 (3 $3 \sigma^{7} 5$ 우 ㅇ MT; $1 \sigma^{7}$ YT), 4.IX.2000 (1 주 2 우 오 MT; $1 \sigma^{7} 1$ 우 YT), 18.IX.2000 (4 $4 \sigma^{7} \sigma^{7} 2$ 우 우 MT), 25.IX.2000 ( $2 \sigma^{7} \sigma^{\pi} 2$ 우 우 MT). - Barranco de Las Traves: 23.VIII.1999 (1 $\sigma^{7} 1$ ᄋ YT), 3.IV.2000 (1 $\sigma^{\pi}$ MT), 24.IV.2000 (2우 우 MT), 30.V.2000 (3우 우 MT), 5.VI.2000 (5 우 우 MT), 13.VI.2000 (7 $7 \sigma^{\pi} \sigma^{\prime \prime} 12$ 우 우 MT; 2 우 우 YT), 19.VI.2000 (1 5 우 우 MT), 27.VI.2000 (50 $0^{7} 8$ 우 우 MT; 3 우 우 YT), 4.VII.2000 ( $40^{\pi} \sigma^{\pi} 2$ 우 우 MT; $10^{\pi} 1$ 우 YT), 17.VII.2000 (10 1 우 MT; $2 \sigma^{7} \sigma^{7} \mathrm{YT}$ ), 24.VII.2000 (1 $\sigma^{7} 5$ 우 우 MT; $2 \sigma^{\pi} \sigma^{7} \mathrm{YT}$ ),

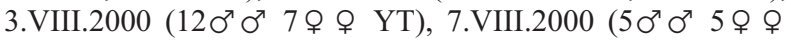
MT; 1 우 YT), 14.VIII.2000 (2우우 MT), 22.VIII.2000

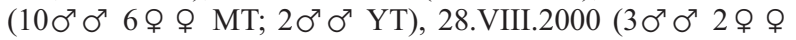
MT; 1 우 YT), 18.IX.2000 (10 2 우 우 MT; $1 \sigma^{\pi}$ YT). - Lomo de las Chozas: 26.IV.2000 (1 ᄋ MT), 1.VI.2000 (2 $\sigma^{\pi} \sigma^{\pi} 1$ ㅇ MT), 21.VI.2000 (4 $\sigma^{\top} \sigma^{\top} 2$ 우 우 MT), 16.VIII.2000 (1 우 MT). - Roque de la Cumbrecita: 23.II.2000 (1 ㅇ MT), 8.III.2000 (10 1 우 MT), 22.III.2000 (1 $1 \sigma^{7} 3$ 우 우 MT), 5.IV.2000 (1 우 YT), 26.IV.2000 (1 $1 \sigma^{\top} 3$ 우 우 MT; $\left.1 \sigma^{\top} \mathrm{YT}\right), 10 . \mathrm{V} .2000$ (1 $1 \sigma^{\top}$ 3 우 우 MT; 2 우 우 YT), 24.V.2000 (1 우 MT), 1.VI.2000

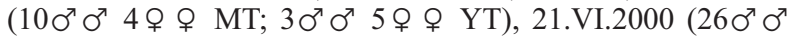

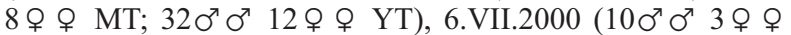

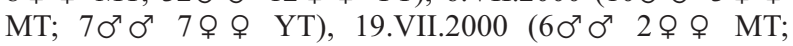

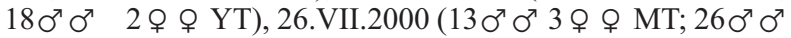

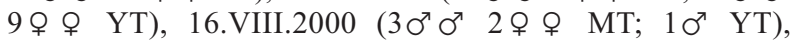

24.VIII.2000 (1 \& MT; $10^{7} \quad$ YT), 30.VIII.2000 (1 \& YT), 6.IX.2000 ( $2 \sigma^{7} \sigma^{7} 1$ \& YT). - Veta de Tajodeque: 17.VI.2000 (1 $\sigma^{7}$ on flowers of Argyranthemum haouarytheum). - Roque de los Muchachos: 21.III.2001 (1우 MT; 2 우 우 YT), 28.III.2001 (1 우 YT), 10.IV.2001 (2우 우 MT; $10^{\pi} 1$ 우 YT), 25.IV.2001 (2우 우 MT), 9.V.2001 (1 우 MT), 17.V.2001 (10 $\mathrm{MT}), 23 . \mathrm{V} .2001$ (1 ᄋ MT), 31.V.2001 (20 $\sigma^{\pi} 1$ 우 $\left.\mathrm{MT}\right)$, 4.VII.2001 (10 MT).

Distribution. Endemic to the Canary Islands (Tenerife, Gran Canaria, La Gomera, La Palma).

Hosts. Unknown. Other species of Siphona parasitize either larvae of Tipulidae (Diptera) or Noctuidae and Geometridae (Lepidoptera).

REMARKS. The body length of $S$. seyrigi varies between 2 and $4 \mathrm{~mm}$ (the majority between 2 and $3 \mathrm{~mm}$ ). The number of postsutural dorsocentral bristles is normally 3 , but can be 4 in larger specimens.

\section{Subfamily Dexiinae}

Estheria simonyi (Brauer \& Bergenstamm, 1891)

Material (Total 3 MT). Playa de Taburiente: 8.V.2000 (1 ㅇ MT), 27.VI.2000 (1 9 MT). - Barranco de Las Traves: 24.IV.2000 (1 $\left.0^{7} \mathrm{MT}\right)$.

Distribution. Endemic to the Canary Islands (Tenerife, Gran Canaria, La Palma, El Hierro).

Hosts. Unknown.

\section{Campylocheta inepta (Meigen, 1824)}

Material (Total 14 MT, 3 YT). Playa de Taburiente: 8.XI.1999 (1 ㅇ MT), 7.II.2000 (1 ㅇ MT), 6.III.2000 (3우 우 MT), 3.IV.2000 (1 ㅇ YT), 24.IV.2000 (1 우 MT), 8.V.2000 (1 ㅇ YT), 30.V.2000 (20 $\sigma^{7}$ MT), 5.VI.2000 (30 $\sigma^{7}$ MT), 27.VI.2000 (20 $\sigma^{\pi}$ MT), 18.IX.2000 (1 $\circ$ MT). - Roque de los Muchachos: 23.V.2001 (1 우 YT).

Distribution. Europe, Transcaucasia, southern Siberia. - Canary Islands: Tenerife, La Palma, El Hierro.

Hosts. Numerous Geometridae, occasionally also some other Macrolepidoptera. Krauss (1964) records $C$. inepta from the Canary Islands (Tenerife) as reared from an unidentified Geometridae.

\section{Ramonda prunicia (Herting, 1969)}

Material (Total 51 MT, 235 YT). Playa de Taburiente: 3.I.2000 (1 우 MT), 3.IV.2000 (1 우 MT), 24.IV.2000 (1 우 MT). - Barranco de Las Traves: 10.VII.2000 (10 MT), 24.VII.2000 (10 MT). - Lomo de las Chozas: 8.XII.1999 (1 우 MT), 23.II.2000 (1 우 MT; 3 우 우 YT), 8.III.2000 (2 $\sigma^{7} \sigma^{\pi}$

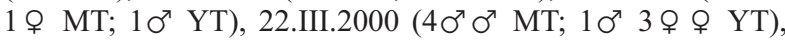

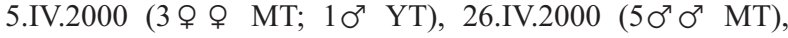


10.V.2000 (3 $3 \sigma^{\pi} \sigma^{\pi}$ MT), 24.V.2000 (1 ㅇ MT), 21.VI.2000 (1 $\sigma^{\pi}$ YT). - Roque de la Cumbrecita: 24.XI.1999 (1 우 MT), 5.I.2000 (1 ㅇ MT), 9.II.2000 (1 $\sigma^{\top}$ MT), 22.III.2000 (1 $\sigma^{7} 1$ ㅇ MT), 5.IV.2000 (1 \& MT; $\left.1 \sigma^{7} \mathrm{YT}\right), 26 . \mathrm{IV} .2000$ (2 $\left.\sigma^{7} \sigma^{7} \mathrm{MT}\right)$, 10.V.2000 (1 ○َ MT; $1 \sigma^{7}$ YT), 1.VI.2000 (1 ᄋ YT), 6.VII.2000 (1 $\sigma^{7}$ YT), 16.VIII.2000 (1 ㅇ MT). - Roque de los Muchachos:

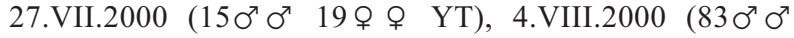
39 우 우 YT), 25.VIII.2000 (4ه $\sigma^{\top} 2$ 우 우 YT), 31.VIII.2000 ( $20^{\pi} 0^{\top} 7$ 우 우 YT), 7.IX.2000 (3우 우 YT), 14.IX.2000 (8우 우 YT), 21.IX.2000 (5우 우 YT), 28.IX.2000 (2우 우 YT), 4.X.2000 (1 우 MT; $1 \sigma^{\top} 3$ 우 우 YT), 11.X.2000 (5우 우 MT), 17.X.2000 (1 ㅇ YT), 10.IV.2001 (1 우 YT), 2.V.2001 (1 우 YT), 17.V.2001 (1 ㅇ YT), 23.V.2001 (2 우 우 YT), 31.V.2001 (1 $\sigma^{T} 4$ 우 우 YT), 6.VI.2001 (1 $1 \sigma^{7} 1$ 우 YT), 14.VI.2001 (1 $\sigma^{\pi}$ MT; $10^{\pi}$ YT), 20.VI.2001 (2 $\sigma^{7} \sigma^{\top} \mathrm{MT} ; 1 \sigma^{\top} 3$ 우 우 YT), 28.VI.2001 (2

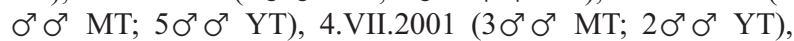
11.VII.2001 (10 MT; $20^{\pi} \sigma^{\pi} 1$ Q Y YT).

Distribution. Palaearctic. - Canary Islands: La Palma.

Hosts. Several Noctuidae (Lepidoptera). No host records from the Canary Islands.

\section{Rondania insularis (Bigot, 1891)}

MAterial (Total 93 MT, 26 YT). Playa de Taburiente: 18.X.1999 (1 ᄋ MT), 15.XI.1999 (1 $\sigma^{\pi} 1$ ㅇ MT), 7.II.2000 (3

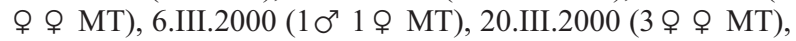
7.VIII.2000 (4 $\sigma^{7} \sigma^{7}$ MT). - Lomo de las Chozas: 22.IX.1999

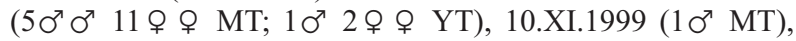
9.II.2000 (1 $10^{7} 1$ 우 MT), 23.II.2000 (1 우 MT), 8.III.2000 (1 $\sigma^{\prime \prime}$ MT; 1 우 YT), 1.VI.2000 (1 우 MT). - Roque de la Cumbrecita: 10.XI.1999 (1 우 MT), 21.VI.2000 (1 주 YT), 26.VII.2000 (1 우 MT). - Roque de los Muchachos: 27.VII.2000 (1 $\sigma^{7}$ MT; $1 \sigma^{7} 1$ 우 YT), 4.VIII.2000 (130 $\sigma^{\pi} 7$ 우 우 MT; $80^{7} \sigma^{\pi} 6$ 우 우 YT),

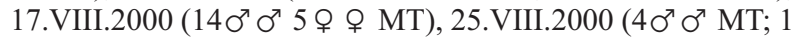
○ 1 우 YT), 21.IX.2000 (1 $\left.\sigma^{\pi} \mathrm{YT}\right), 2$ 28.III.2001 (1 우 YT), 2.V.2001 (1 우 YT), 23.V.2001 (1 우 MT), 31.V.2001 (1 우 MT), 6.VI.2001 (1 우 MT), 14.VI.2001 (2우 우 MT), 20.VI.2001 (1 우 $\mathrm{MT}), 28 . \mathrm{VI} .2001$ (10 1 우 $\mathrm{MT}), 4 . \mathrm{VII} .2001$ (1우 $\mathrm{MT}$ ), 15.VII.2001 (1 오 MT).

Distribution. Endemic to the Canary Islands (Tenerife, Gran Canaria, La Gomera, La Palma).

Hosts. Unknown. Other species of Rondania develop in adult Curculionidae (Coleoptera).

\section{Subfamily Phasiinae}

Phasia pusilla Meigen, 1824

Material (Total 8 MT, 1 YT). Barranco de Las Traves: 5.VI.2000 (1 $\sigma^{7}$ MT; $1 \sigma^{7}$ YT), 13.VI.2000 (4の $\sigma^{7}$ MT),

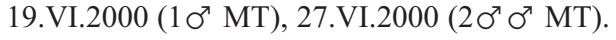
Palma.

Distribution. Palaearctic. - Canary Islands: La

Hosts. Some Lygaeidae, Cydnidae and Anthocoridae (Hemiptera). No host record from the Canary Islands.
Eulabidogaster setifacies (Rondani, 1861)

MAterial (Total 2 MT). Barranco de Las Traves: 27.VI.2000 (1 ㅇ MT), 4.VII.2000 (1 우 MT).

Distribution. Warmer parts of the Palaearctic, except East Asia. - Canary Islands: La Palma.

Hosts. Only a single host record (from Israel) known up to the present: Corizus hyoscyami Linnaeus (Hemiptera, Coreidae).

REMARKS. Before the present investigation there was only one record from La Palma, almost one hundred years old (Becker, 1908).

\section{Cylindromyia brassicaria (Fabricius, 1775)}

MAterial (Total 6 MT, 2 YT). Barranco de Las Traves: 5.IX.1999 (10 MT), 4.VII.2000 (1 우 MT), 17.VII.2000 (3 $\sigma^{\top} \sigma^{\top}$ MT). - Roque de los Muchachos: 14.IX.2000 (2 우 우 YT), 15.VII.2001 (1 ㅇ MT).

Distribution. Palaearctic. - Canary Islands: Tenerife, Gran Canaria, La Palma.

Hosts. Dolycoris spp., Holcostethus vernalis Wolff (Hemiptera, Pentatomidae). No host record from the Canary Islands.

REMARKS. The abdominal tergites 2 and 3 of the La Palma-population of $C$. brassicaria are entirely or predominantly black dorsally, but it must be a simple colour variation, because no other differences can be found, either in the exterior morphology or in the genitalia of males and females.

\section{Phenology}

The phenology of the species is shown in Table 1. Most species are bivoltine or plurivoltine but can perhaps also be univoltine in case they undergo their larval development at higher altitudes. The seasonal peak in summer (June, July or August) is most probably due to the fact that most material has been collected at higher altitudes. A univoltine species which flies only in spring is Gonia quadrisetosa. Judging from published and unpublished data from other islands of the Canarian Archipelago, Estheria simonyi could also be univoltine, because no data are known for this species from August to December.

\section{Altitude}

Table 2 shows how many specimens of tachinids were collected at different altitudes. The fact that some species were predominantly collected at 
Table 1.- Number of tachinid specimens in the respective month in which the traps were emptied.

Tabla 1.- Ejemplares de taquínidos en el mes de la recogida del material.

\begin{tabular}{|c|c|c|c|c|c|c|c|c|c|c|c|c|}
\hline & JAN & FEB & MAR & APR & MAY & JUN & JUL & AUG & SEP & OCT & NOV & DEC \\
\hline Exorista segregata & 1 & 1 & 2 & 1 & 2 & 1 & 2 & 19 & 10 & 4 & 2 & 2 \\
\hline Exorista sorbillans & & & & & & & & 1 & & & & \\
\hline Exorista kugleri & & & & & & & & 1 & 1 & & & \\
\hline Chetogena acuminata & & & & 2 & 3 & 29 & 13 & 42 & 11 & 2 & 1 & \\
\hline Ceracia mucronifera & & & & 1 & 1 & 6 & 12 & 56 & 24 & 1 & 1 & \\
\hline Nemorilla maculosa & & & 1 & & & 3 & 7 & 40 & 6 & 4 & & \\
\hline Aplomya confinis & & & & 1 & 1 & 22 & 5 & 7 & & & & \\
\hline Pales cyanea & & & 1 & 2 & 4 & & & 16 & 4 & & & \\
\hline Gonia quadrisetosa & & & 1 & 3 & 1 & & & & & & & \\
\hline Pseudogonia fasciata & & & & 1 & 1 & & 1 & 1 & 4 & & 1 & \\
\hline Tachina canariensis & & & 1 & 4 & 4 & 3 & 2 & 3 & 2 & 1 & 1 & \\
\hline Linnaemya soror & & & & 1 & & 2 & 1 & & & & & \\
\hline Macquartia tessellum & & 1 & 1 & & & 2 & 1 & 2 & & & & \\
\hline Phytomyptera vaccinii & & & 3 & & & & 2 & 3 & & & & \\
\hline Siphona seyrigi & 2 & 15 & 21 & 69 & 74 & 174 & 130 & 94 & 22 & 2 & & \\
\hline Estheria simonyi & & & & 1 & 1 & 1 & & & & & & \\
\hline Campylocheta inepta & & 1 & 3 & 2 & 4 & 5 & & & 1 & & 1 & \\
\hline Ramonda prunicia & 2 & 5 & 14 & 16 & 15 & 19 & 46 & 138 & 18 & 11 & 1 & 1 \\
\hline Rondania insularis & & 6 & 8 & & 3 & 8 & 6 & 63 & 20 & 1 & 4 & \\
\hline Phasia pusilla & & & & & & 9 & & & & & & \\
\hline Eulabidogaster setifacies & & & & & & 1 & 1 & & & & & \\
\hline Cylindromyia brassicaria & & & & & & & 5 & & 3 & & & \\
\hline Total & 5 & 29 & 56 & 104 & 114 & 285 & 234 & 486 & 126 & 26 & 12 & 3 \\
\hline
\end{tabular}

a certain altitudinal belt is not proof that they really undergo their larval development in that zone. Most Tachinidae are good flyers and specimens from the coastal areas of the island could easily fly long distances up to high altitudes in summer. Some of the species from La Palma (Aplomya confinis, Linnaemya soror, Macquartia tessellum) are known to have hilltopping behaviour (see Tschorsnig, 1996). Also specimens of Exorista sorbillans, E. kugleri, Chetogena acuminata, Ceracia mucronifera and Tachina canariensis were found to be hilltopping during a collecting trip by the first author to Tenerife in 1996 (unpublished).

\section{Discussion}

Only one species (Gonia bimaculata Wiedemann, 1819), which was known before from La Palma (from "los Llanos" and "La Caldera", compare Báez et al., 1986), was not collected during the present investigation, so the total number of tachinid species on La Palma is 23 . They represent $43 \%$ of the total Canarian tachinid fauna of 54 species (52 from Báez et al., 1986, one additional record in Tschorsnig \& Báez, 2002, and one unpublished record). Three of them (Ramonda prunicia, Phasia pusilla, Eulabidogaster setifacies) are only known from La Palma within the Canary Islands. Five species (Pales cyanea, Gonia quadrisetosa, Tachina canariensis, Siphona seyrigi, Rondania insularis) are endemic to the Canary Islands.

The spectrum and number of species depends on collecting methods. Direct samples with a butterfly net (from stones, leaves, flowers etc.) would yield a different spectrum than Malaise trap or yellow pan trap samples. The Malaise traps (799 specimens) turned out to be only slightly more effective than the yellow pan traps (679 specimens) in respect to the number of specimens, but both types collected the same number of species (20). This is, however, only an average value, because different species may have a clear preference for one of the types (compare total numbers in the species list).

Malaise traps are normally especially effective for the collection of small or very small species of 
Table 2.- Number of tachinid specimens collected at different altitudes.

Tabla 2.- Ejemplares de taquínidos recolectados en diferentes altitudes.

\begin{tabular}{|c|c|c|c|c|c|}
\hline & $750 \mathrm{~m}$ & $1068 \mathrm{~m}$ & $\begin{array}{c}1277 \\
(-1297 \mathrm{~m})\end{array}$ & 1377 m & $2250 \mathrm{~m}$ \\
\hline Exorista segregata & 16 & 7 & 18 & 5 & 1 \\
\hline Exorista sorbillans & 1 & & & & \\
\hline Exorista kugleri & & 1 & 1 & & \\
\hline Chetogena acuminata & 10 & 1 & 44 & 11 & 37 \\
\hline Ceracia mucronifera & 9 & 71 & 1 & 21 & \\
\hline Nemorilla maculosa & 18 & 14 & & 3 & 26 \\
\hline Aplomya confinis & 1 & 28 & 1 & 2 & 4 \\
\hline Pales cyanea & 10 & & 3 & 2 & 12 \\
\hline Gonia quadrisetosa & & & & & 5 \\
\hline Pseudogonia fasciata & 2 & & & 1 & 5 \\
\hline Tachina canariensis & 4 & & 1 & & 16 \\
\hline Linnaemya soror & 1 & & 1 & & 2 \\
\hline Macquartia tessellum & & 1 & 1 & & 5 \\
\hline Phytomyptera vaccinii & 8 & & & & \\
\hline Siphona seyrigi & 200 & 136 & 11 & 238 & 17 \\
\hline Estheria simonyi & 2 & 1 & & & \\
\hline Campylocheta inepta & 16 & & & & 1 \\
\hline Ramonda prunicia & 3 & 2 & 31 & 14 & 236 \\
\hline Rondania insularis & 15 & & 26 & 3 & 75 \\
\hline Phasia pusilla & & 9 & & & \\
\hline Eulabidogaster setifacies & & 2 & & & \\
\hline Cylindromyia brassicaria & & 5 & & & 3 \\
\hline Total & 316 & 278 & 139 & 300 & 445 \\
\hline
\end{tabular}

Diptera. Therefore it was surprising that the large species Tachina canariensis was more commonly caught in the Malaise traps than in the yellow pan traps.

Yellow pan traps were clearly more effective than Malaise traps at high altitudes. The ratio (given as a percentage) of number of specimens caught in yellow pan traps to total number of specimens (caught in Malaise traps + yellow pan traps) is as follows for the different altitudes: $750 \mathrm{~m}$ : 20\%; $1068 \mathrm{~m}: 25 \%$; 1277[-1297] m: 45\%; $1377 \mathrm{~m}$ : $50 \% ; 2250 \mathrm{~m}: 75 \%$. This is most probably due to the fact that yellow pan traps are more effective in cold weather (which prevails at high altitudes - or in the early or late season). This effect has already been observed for many years by the first author (for Tachinidae) at several localities, but a sound scientific explanation is still missing for it.

The most common species found in this investigation was the small Siphona seyrigi (603 specimens), which is also common on other islands of the Canarian Archipelago (Tenerife, Gran Canaria, La Gomera).

It would be of special interest to determine the hosts of tachinid species on La Palma, because nothing is known about host relations from this island.

\section{References}

BÁez, M., Herting, B. \& Tschorsnig, H.-P., 1986. The Tachinidae (Diptera) of the Canary Islands. Stuttgarter Beiträge zur Naturkunde, Serie A (Biologie), 394: 1-15.

Domingo-Quero, T., Alonso-Zarazaga, M. A., Sánchez-Ruiz, A., Araujo Armero, R., Navas Sánchez, A., SÁnchez Moreno, S., García Becerra, R., Nebreda, M., SÁnchez Ruiz, M., Fontal-CAZAlla, F. \& Nieves-Aldrey, J. L., 2003. Inventariando la biodiversidad en el Parque Nacional de la Caldera de Taburiente (La Palma, Islas Canarias, España): novedades científicas. Graellsia, 59(2-3): 45-68. 
Enderlein, G., 1930. Entomologica Canaria. VII. Zoologischer Anzeiger, 92: 41-56.

Herting, B. \& Dely-Draskovits, A., 1993. Family Tachinidae. In: Soós, A. \& Papp, L. (eds.). Catalogue of Palaearctic Diptera, 13: 118-624.

KrAUSS, N. L. H., 1964. Insects associated with firebush (Myrica faya Aiton). Proceedings of the Hawaiian entomological Society, 18: 405-411.

LeIPNitZ, M. \& Tschorsnig, H.-P., 2005. Neue oder interessante Wirtsbefunde von europäischen Raupenfliegen (Diptera: Tachinidae). Mitteilungen entomologischer Verein Stuttgart, 40: 56.

Mesnil, L. P., 1956. Larvaevorinae (Tachininae). In: Lindner, E. (ed.). Die Fliegen der paläarktischen Region, 64g: 513-554.

TsCHORSNIG, H.-P., 1996. Gipfelbesuchende Raupenfliegen (Diptera, Tachinidae) in Westeuropa. Mitteilungen des internationalen entomologischen Vereins Frankfurt am Main, 21: 1-19.

Tschorsnig, H.-P., in press. Tachinidae. In: Baur, H. (ed.). Determination list of entomophagous insects, Nr. 14. International Organization for biological and integrated control of noxious Animals and Plants/West Palaearctic regional Section Bulletin.
Tschorsnig, H.-P. \& BÁEz, M., 2002. Tachinidae. In: Carles-Tolrá Hjorth-Andersen, M. (coord.). Catálogo de los Diptera de España, Portugal y Andorra (Insecta). Monografías de la Sociedad entomológica aragonesa 8: 225-234.

Tschorsnig, H.-P. \& Herting, B., 1997. Tachinidae. In: Vidal, S. (Ed.). Determination list of entomophagous insects, Nr. 13. International Organization for biological and integrated control of noxious Animals and Plants/West Palaearctic regional Section Bulletin, 20: $28-38$
Recibido, 14-XII-2006 Aceptado, 12-IV-2007

Publicado, 27-VI-2007 